Article

\title{
Long-Term Effects of Spironolactone on Kidney Function and Hyperkalemia-Associated Hospitalization in Patients with Chronic Kidney Disease
}

\author{
Chen-Ta Yang ${ }^{1,+}$, Chew-Teng Kor ${ }^{1,+}$ and Yao-Peng Hsieh 1,2,3,* \\ 1 Department of Internal Medicine, Changhua Christian Hospital, Changhua 50006, Taiwan; \\ 149054@cch.org.tw (C.-T.Y.); 179297@cch.org.tw (C.-T.K.) \\ 2 School of Medicine, Kaohsiung Medical University, Kaohsiung 80708, Taiwan \\ 3 School of Medicine, Chung Shan Medical University, Taichung 40201, Taiwan \\ * Correspondence: 102407@cch.org.tw; Tel.: +886-4-7238595 (ext. 5889); Fax: +886-4-7277982 \\ + These authors contributed equally to this work.
}

Received: 14 November 2018; Accepted: 19 November 2018; Published: 21 November 2018

check for updates

\begin{abstract}
Background: Spironolactone, a non-selective mineralocorticoid receptor antagonist, can protect against cardiac fibrosis and left ventricular dysfunction, and improve endothelial dysfunction and proteinuria. However, the safety and effects of spironolactone on patient-centered cardiovascular and renal endpoints remain unclear. Methods: We identified predialysis stage 3-4 chronic kidney disease (CKD) patients between 2000 and 2013 from the Longitudinal Health Insurance Database 2005 (LHID 2005). The outcomes of interest were end-stage renal disease (ESRD), major adverse cardiovascular events (MACE), hospitalization for heart failure (HHF), hyperkalemia-associated hospitalization (HKAH), all-cause mortality and cardiovascular mortality. The Fine and Gray sub-distribution hazards approach was adopted to adjust for the competing risk of death. Results: After the propensity score matching, 693 patients with stage 3-4 CKD were spironolactone users and 1386 were nonusers. During the follow-up period, spironolactone users had a lower incidence rate for ESRD than spironolactone non-users (39.2 vs. 53.69 per 1000 person-years) and a higher incidence rate for HKAH (54.79 vs. 18.57 per 1000 person-years). The adjusted hazard ratios for ESRD of spironolactone users versus non-users were 0.66 (95\% CI, $0.51-0.84 ; p$ value $<0.001)$ and 3.17 (95\% CI, 2.41-4.17; $p$ value < 0.001) for HKAH. A dose-response relationship was found between spironolactone use and risk of ESRD and HKAH. There were no statistical differences in MACE, HHF, all-cause mortality and cardiovascular mortality between spironolactone users and non-users. Conclusion: Spironolactone represented a promising treatment option to retard CKD progression to ESRD amongst stage 3-4 CKD patients, but strategic treatments to prevent hyperkalemia should be enforced.
\end{abstract}

Keywords: chronic kidney disease (CKD); end-stage renal disease (ESRD); major adverse cardiovascular events (MACE); mortality; spironolactone

\section{Introduction}

Chronic kidney disease (CKD), having an increasing prevalence rate of about $10 \%$ worldwide as a result of longevity and ongoing epidemic of diabetes mellitus (DM), is an emerging global health concern and associated with the risk of premature cardiovascular disease (CVD) and mortality $[1,2]$. The above-expected cardiovascular risk may be attributed to some unique features in CKD, comprising anemia, endothelial dysfunction, oxidative stress, abnormalities in calcium and 
phosphorus metabolism and metabolic acidosis. These derangements can further accelerate coronary calcification, coronary atherosclerosis, left ventricular hypertrophy and eventually heart failure [3].

Among the available treatment options to retard renal function decline, blockade of renin-angiotensin-aldosterone system (RAAS) by angiotensin-converting enzyme inhibitor (ACEI) or angiotensin receptor blocker (ARB) has been widely used for their potentially beneficial effects on renal and cardiovascular outcomes in both diabetic and non-diabetic patients $[4,5]$. The renoprotective impact takes effects mainly as a result of reduction of intra-glomerular pressure and inhibition of angiotensin-II induced mesangial cell proliferation and fibrosis [6]. The phenomenon of aldosterone synthesis escape had been proposed to explain that neither ACEI nor ARB can completely abrogate or retard the progression of kidney disease [7]. Therefore, mineralocorticoid receptor antagonists (MRA) have been evaluated for their promising role in conjunction with ACEI or ARB afterwards.

Several studies on the effects of MRA were conducted amongst CKD patients in the past one decade. A 2014 meta-analysis, updated from a 2009 meta-analysis, included 1549 patients of 27 studies and demonstrated that addition of MRA to ACEI or ARB (or both) in mild to moderate CKD patients resulted in the reduction of proteinuria and blood pressure, but precluded conclusive evidence regarding the risk of major cardiovascular events or end-stage renal disease (ESRD) [8]. The higher risk of hyperkalemia and gynecomastia limits its prescription in clinical practice. A later meta-analysis by $\mathrm{Ng}$ et al. in 2015 also drew similar conclusions, but the impact of MRA on CVD morbidity and mortality was unable to evaluate due to insufficiency data [9]. The major flaws in the studies of these meta-analyses were relatively small patient numbers and short follow-up period relatively. Spironolactone, a non-selective MRA, can protect against cardiac fibrosis and left ventricular dysfunction, and improve endothelial dysfunction and proteinuria [10]. Therefore, we conducted this retrospective cohort study using representative national data to evaluate the effects of spironolactone on all-cause mortality, CVD mortality, ESRD, hospitalization for heart failure (HHF), major adverse cardiovascular events (MACE) and hyperkalemia-associated hospitalization (HKAH) in predialysis stage $3-4$ CKD patients.

\section{Experimental Section}

\subsection{Data Source}

The National Health Insurance Research Database (NHIRD) of Taiwan, which included the healthcare utilization data covering $99 \%$ of Taiwanese population, was released for the purpose of scientific research. The Longitudinal Health Insurance Database 2005 (LHID 2005), which contained the information of 1 million people, was randomly selected from the NHIRD and was used as the research database for our study. LHID 2005 contained the comprehensive de-identified healthcare information on demographic, outpatient visit, inpatient care, prescription drugs and medical procedures from 1996 to 2013. The Bureau of Taiwan Health Insurance adopted the International Classification of Diseases, Ninth Revision, Clinical Modification (ICD-9-CM) codes for medical coding. The drugs were classified according to the Anatomical Therapeutic Chemical (ATC) classification system. This study was approved by the Institutional Review Board (IRB) of the Changhua Christian Hospital (approval number CCH-IRB 180717) and conducted in accordance with the declaration of Helsinki. The written informed consents were waived for a retrospective study in Taiwan.

\subsection{Study Design and Participants}

From 1996 to 2013, we identified 21,693 patients with CKD who were defined as having at least one record of a CKD diagnostic code made by a nephrologist per month for at least three consecutive months. We used a 4-year look-back (1996-1999) period to determine patients with incident CKD by excluding pre-existing CKD diagnosis. In addition, incident CKD patients who had incomplete demographic data; were aged $<18$ years, $>100$ years; had advanced CKD (stage 5), acute kidney injury, end-stage renal disease; or had a follow-up time of $<90$ days were also excluded. The remaining 
incident CKD patients represented moderate to severe (stage 3-4) CKD patients. Among them, patients who had received spironolactone within 90 days after CKD diagnosis were considered as spironolactone users, whereas the remaining patients were considered as non-users. Because we used drug prescription information within 90 days after CKD diagnosis to ascertain spironolactone use, the 91st day after CKD diagnosis was defined as the index date.

\subsection{Outcome Measures and Relevant Variables}

The outcomes of interest were ESRD requiring renal replacement therapy, MACE, HHF, HKAH, all-cause mortality and CVD mortality. MACE was the composite of acute myocardial infarction (ICD-9 code 410) and ischemic stroke (ICD-9 code 433-436). We defined HHF and HKAH as the first listed ICD-9 code 428 or 276.7 of the discharge diagnoses during the follow-up period, respectively. Causes of death were defined by either the main diagnosis for in-hospital death or the first discharge diagnosis of the last hospitalization within three months before death outside the hospital. The end of study was on 31 December 2013. Death was considered a competing event for the occurrence of ESRD, MACE, HHF, and HKAH.

Potential relevant confounders included demographic (age, gender, monthly income and geographic location), comorbid diseases defined by at least three corresponding diagnostic codes within 1 year before index date, and long-term medications use, such as anti-hypertensive drugs, anti-diabetic medication, statin, aspirin, NSAIDs and NaHCO3. Charlson comorbidity index scores (CCIs) were used to measure the severity of baseline comorbidities.

\subsection{Statistical Analyses}

Data were presented as mean \pm standard deviation (SD) for continuous variables and number (percentage) for categorical variables. The distribution of patients' characteristics between spironolactone users and non-users was compared using chi-squared tests and t-test for categorical and continuous variables, respectively. The propensity score was calculated by performing non-parsimonious multivariate logistic regression using all the patient's characteristic variable (Table 1). The resulting propensity score indicated the probability of being treated with spironolactone and was used for matching processes. The spironolactone non-users were matched by propensity score to the spironolactone users in 2:1 ratio. We used the nearest-neighbor algorithm with a caliper of 0.1 standard deviation (SD) units to construct matched pairs, with the assumption that the proportion of 1.0 is perfect.

We calculated the incidence rate (per 1000 person-years) for outcomes of interest. The cumulative incidence of study outcomes over time between spironolactone users and non-users was estimated using modified Kaplan-Meier method (Fine and Gray sub-distribution hazards approach) and compared using Gray's tests. The Cox proportional hazards regression models were performed to assess the association between spironolactone use and outcomes of interest, with hazard ratio (HR) and $95 \%$ confidence interval (CI) to determine the statistical significance. Because death would impede the occurrence of other study outcomes, the Fine and Gray sub-distribution hazards approach was adopted to adjust for the competing risk of death.

To determine the dose-response association, we estimated the risk of clinical outcomes for spironolactone users versus non-users according to the prescribed daily dose $(<12.5,12.5-25$, or $\geq 25 \mathrm{mg}$ ) and the cumulative defined daily dose (DDD) during the 90-day exposure period ( $\leq 30$ or $>30 \mathrm{DDD}$ ). The spironolactone DDD defined by WHO is $75 \mathrm{mg}$. Subgroup analyses were performed to assess the effect modification and we tested the association between spironolactone use and study outcomes of statistical significance in different patient groups. Furthermore, we performed a series of sensitivity analyses to obtain a robust result. First, we evaluated misclassification bias by re-defining spironolactone use at interval of 60,120 and 180 days after CKD diagnosis. Second, we excluded patients in control cohort who received spironolactone during the follow-up period. Third, an as-treat (AT) model was performed to examine the results when patients who switched to receive or discontinue spironolactone were censored. Fourthly, we also checked the cohort effects by dividing all the study 
patients into two exposure year periods. Lastly, we re-ran the multivariate regression analysis using the raw data before propensity score matching (spironolactone users $=785$, non-users $=13,884$ ). All statistical analyses were performed using R language and SPSS statistical software, version 20.0 (SAS 9.4 software (SAS Institute Inc., Cary, NC, USA)). Two-tailed $p$-value $<0.05$ was considered as significant.

\section{Results}

\subsection{Characteristics of Patients}

A flowchart of the subjects' selection process was shown in Figure 1. After excluding CKD patients with advanced (stage 5) CKD or dialysis-dependent ESRD, a total of 14,699 patients with moderate to severe CKD (stage 3-4) were enrolled from LHID 2005 between 2000 and 2013. After the propensity score matching in a 1:2 ratio, 693 patients were spironolactone users and 1386 were nonusers. The mean follow-up time for spironolactone users and non-users were $3.57 \pm 3.2$ and $3.24 \pm 3.23$ years, respectively. Table 1 shows the baseline characteristics of study patients stratified by spironolactone use before and after propensity score matching. After matching, there was no significant difference in the distribution of all baseline characteristics between spironolactone users and non-users. The spironolactone users had a reduced prevalence of ESRD $(12.7 \%$ vs. $19.19 \%, p$ value $<0.001)$ but an increased prevalence of HKAH $(17.75 \%$ vs. $6.64 \%, p$ value $<0.001)$ compared with the spironolactone non-users. However, there was no significant difference in prevalence of MACE, HHF and mortality.

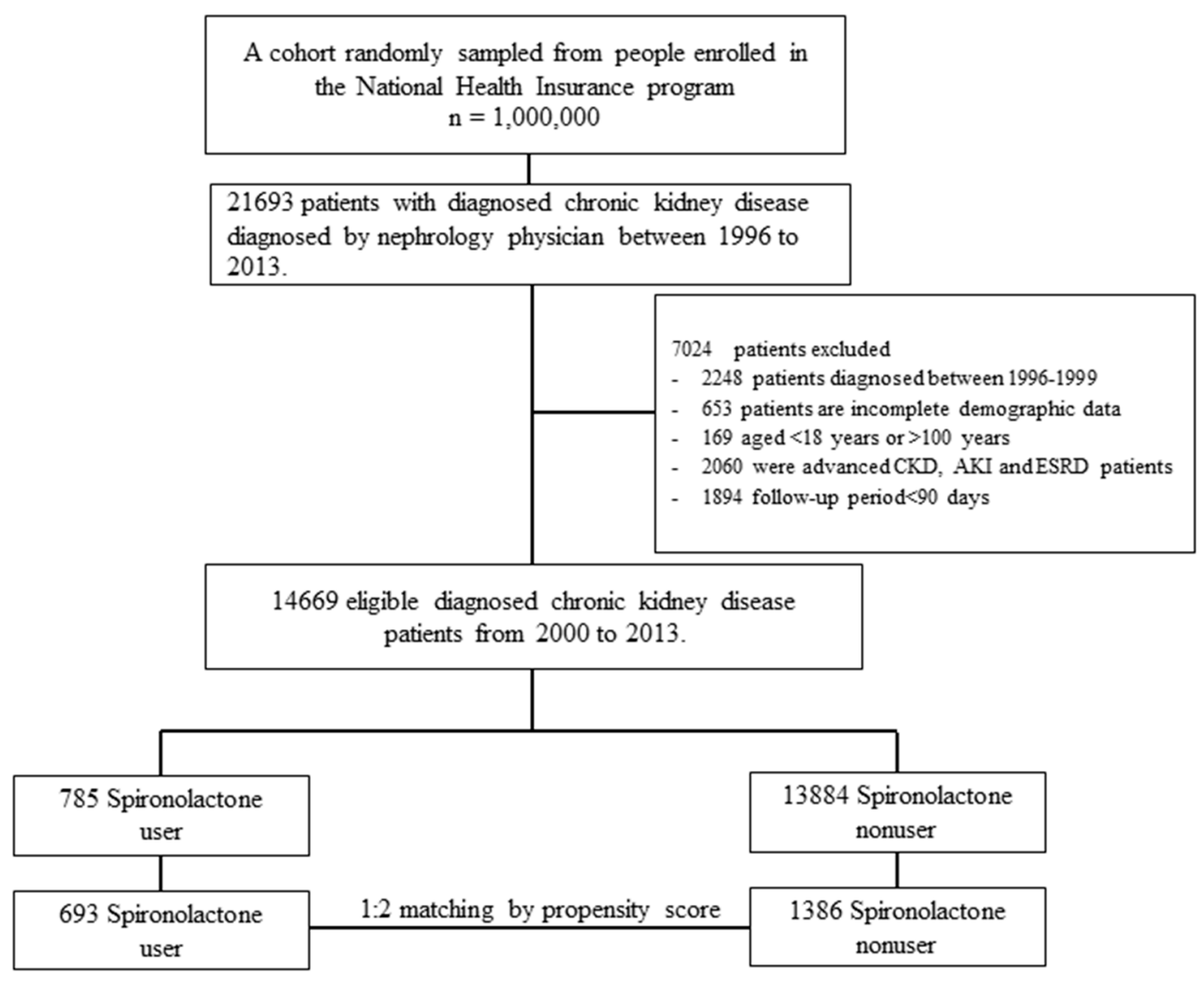

Figure 1. Flowchart of patient selection processes for stage 3-4 chronic kidney disease CKD with or without spironolactone use. 


\subsection{Long-Term Risk of Incident ESRD}

During the follow-up period, spironolactone users had a lower incidence rate for ESRD than spironolactone non-users (39.2 vs. 53.69 per 1000 person-years) (Table 2). Kaplan-Meier curves showed a significantly lower cumulative incidence of ESRD for spironolactone users ( $p$ value $<0.001$ in Figure 2). In Cox's competing risk model analyses, the spironolactone users had a lower risk of ESRD (crude HR, $0.65 ; 95 \%$ CI, $0.51-0.83 ; p$ value < 0.001 ) compared with the spironolactone non-users. After adjustment for all confounders, the association was unchanged (adjusted HR [aHR], 0.66; 95\% CI, 0.51-0.84; $p$ value $<0.001)$. An inverse dose-response relationship was found between spironolactone use and risk of ESRD. Compared with CKD patients not taking spironolactone, patients who took prescribed daily dose of spironolactone $\geq 25 \mathrm{mg}, 12.5-25 \mathrm{mg}$ and $<12.5 \mathrm{mg}$ had significantly a lower risk of ESRD with aHR of $0.57(0.35-0.91), 0.59(0.39-0.91)$, and $0.73(0.52-1.00)$, respectively $(p$ value $=0.0057$ for trend in Table 3). Similarly, those who took cumulative doses of spironolactone $>30$ DDD and $\leq 30$ DDD had a significantly lower risk of ESRD with aHR (95\% CI) of $0.60(0.37-0.97)$ and $0.68(0.52-0.89)$, respectively ( $p$ value $=0.0038$ for trend).

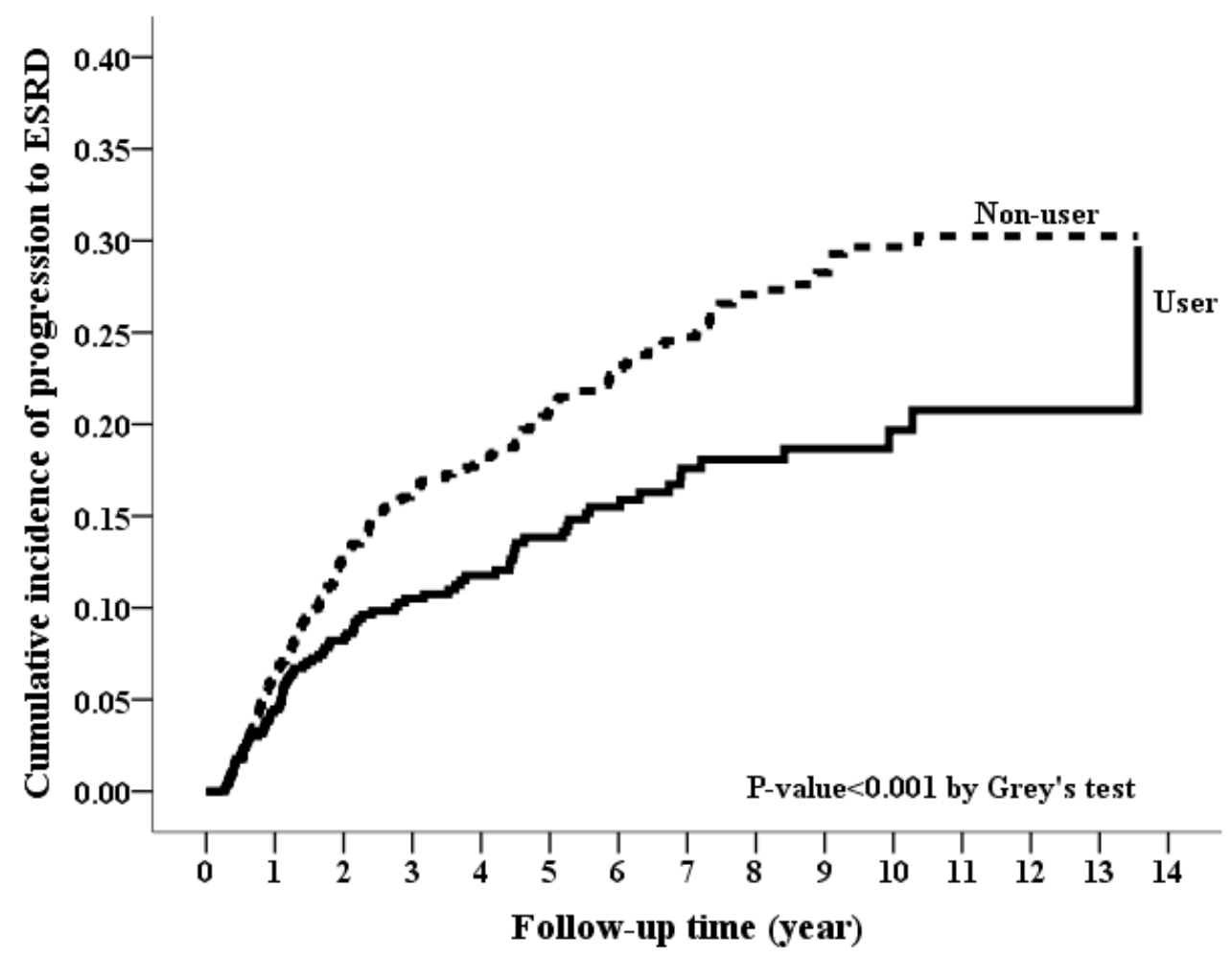

Figure 2. Cumulative incidence rate of progression to end-stage renal disease between spironolactone users and non-users. ( $p$-value $<0.001$, Grey's test).

\subsection{Long-Term Risk of Hyperkalemia-Associated Hospitalization}

During the follow-up period, spironolactone users had a higher incidence rate for HKAH than spironolactone non-users (54.79 vs. 18.57 per 1000 person-years) (Table 2). Spironolactone users also had significantly a higher cumulative incidence for HKAH ( $p$ value $<0.001$ in Figure 3$)$. In both thecrude and adjusted models, the spironolactone users still had a significantly higher risk of HKAH (crude HR, 2.98; 95\% CI, 2.28-3.90; $p$ value < 0.001; aHR, 3.17; 95\% CI, 2.41-4.17; $p$ value < 0.001) than spironolactone non-users (Table 2). In the dose-response effect, the higher dose of spironolactone use was consistently associated with a higher risk for HKAH in both of the defined doses (both $p$ values $<0.001$ for trend in Table 3). 


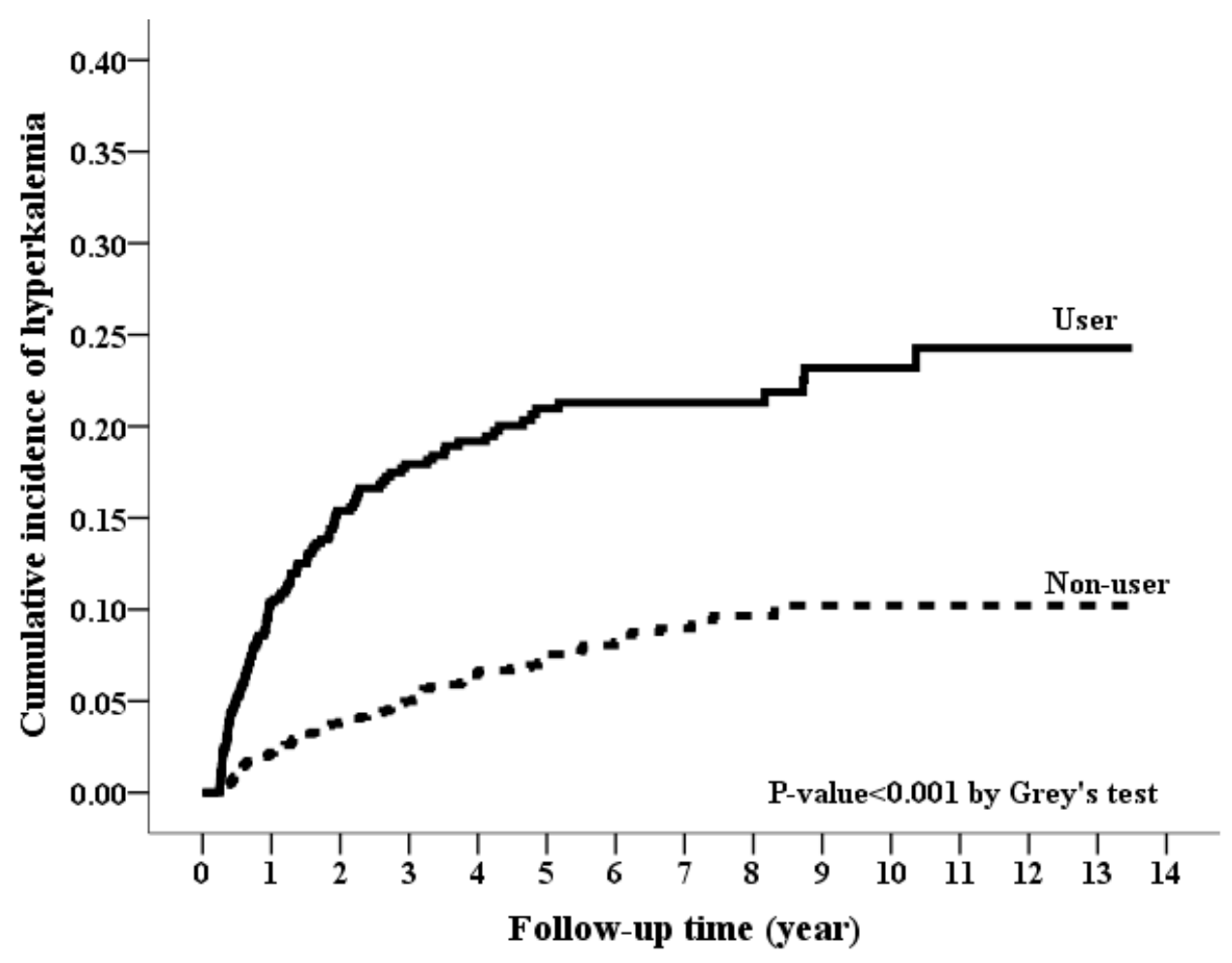

Figure 3. Cumulative incidence rate of hyperkalemia-associated hospitalization between spironolactone users and non-users. ( $p$-value $<0.001$, Grey's test).

\subsection{Long-Term Risk of MACE, Hospitalization for Heart Failure and Mortality}

Kaplan-Meier curves showed non-significant difference in cumulative incidences for MACE, HHF, CVD mortality and all-cause mortality (Figures 4 and 5). Nonetheless, there were no statistically significant differences in the risks of MACE, HHF, CVD mortality, and all-cause mortality between spironolactone users and non-users, both in the crude models and in adjusted models (Table 2). In addition, spironolactone had no dose-response relationship with MACE, HHF, CVD mortality and all-cause mortality (Table 3). 
Table 1. Baseline characteristics and clinical outcomes of study patients by spironolactone use before and after propensity score matching.

\begin{tabular}{|c|c|c|c|c|c|c|}
\hline & \multicolumn{3}{|c|}{ Before Propensity-Score Matching } & \multicolumn{3}{|c|}{ After Propensity-Score Matching } \\
\hline & Non-User & User & $p$-Value & Non-User & User & $p$-Value \\
\hline Patient number & 13,884 & 785 & & 1386 & 693 & \\
\hline Age, years & $63 \pm 16$ & $65 \pm 15$ & $<0.001$ & $65 \pm 15$ & $65 \pm 16$ & 0.814 \\
\hline Gender, Male & $7738(55.73 \%)$ & $440(56.05 \%)$ & 0.862 & 767 (55.34\%) & $380(54.83 \%)$ & 0.827 \\
\hline Monthly income, New Taiwan Dollars & $14,195 \pm 14,667$ & $12,283 \pm 12,489$ & $<0.001$ & $12,419 \pm 12,580$ & $12,397 \pm 12,662$ & 0.971 \\
\hline \multicolumn{7}{|l|}{ Geographic location } \\
\hline Northern & $6661(47.98 \%)$ & $317(40.38 \%)$ & $<0.001$ & $532(38.38 \%)$ & $279(40.26 \%)$ & 0.436 \\
\hline Middle & $2396(17.26 \%)$ & $207(26.37 \%)$ & $<0.001$ & $393(28.35 \%)$ & $180(25.97 \%)$ & 0.274 \\
\hline Southern & $4452(32.07 \%)$ & $248(31.59 \%)$ & 0.813 & $439(31.67 \%)$ & $223(32.18 \%)$ & 0.855 \\
\hline Eastern & $375(2.7 \%)$ & $13(1.66 \%)$ & 0.0968 & $22(1.59 \%)$ & $11(1.59 \%)$ & 0.852 \\
\hline \multicolumn{7}{|c|}{ Comorbidities within 1 year before the index date } \\
\hline Hypertension & 9192 (66.21\%) & $584(74.39 \%)$ & $<0.001$ & $1033(74.53 \%)$ & $514(74.17 \%)$ & 0.859 \\
\hline Diabetes & $5533(39.85 \%)$ & $371(47.26 \%)$ & $<0.001$ & $666(48.05 \%)$ & $326(47.04 \%)$ & 0.664 \\
\hline Coronary artery disease & $2665(19.19 \%)$ & $231(29.43 \%)$ & $<0.001$ & $398(28.72 \%)$ & $195(28.14 \%)$ & 0.783 \\
\hline Stroke & $1924(13.86 \%)$ & $135(17.2 \%)$ & 0.009 & $258(18.61 \%)$ & $114(16.45 \%)$ & 0.225 \\
\hline Atrial fibrillation & $300(2.16 \%)$ & $44(5.61 \%)$ & $<0.001$ & $64(4.62 \%)$ & $34(4.91 \%)$ & 0.770 \\
\hline Cirrhosis & $202(1.45 \%)$ & $72(9.17 \%)$ & $<0.001$ & $74(5.34 \%)$ & $36(5.19 \%)$ & 0.890 \\
\hline PAOD & $267(1.92 \%)$ & $23(2.93 \%)$ & 0.049 & $38(2.74 \%)$ & $21(3.03 \%)$ & 0.709 \\
\hline Cancer & $801(5.77 \%)$ & $57(7.26 \%)$ & 0.083 & $82(5.92 \%)$ & $46(6.64 \%)$ & 0.519 \\
\hline COPD & 2015 (14.51\%) & $166(21.15 \%)$ & $<0.001$ & $298(21.5 \%)$ & $144(20.78 \%)$ & 0.705 \\
\hline CHF & $1359(9.79 \%)$ & $209(26.62 \%)$ & $<0.001$ & $359(25.9 \%)$ & $172(24.82 \%)$ & 0.594 \\
\hline Charlson comorbidity index & $3.1 \pm 2.4$ & $4 \pm 2.6$ & $<0.001$ & $3.8 \pm 2.5$ & $3.8 \pm 2.5$ & 0.826 \\
\hline \multicolumn{7}{|l|}{ Anti-hypertensive drugs } \\
\hline $\mathrm{ACEI} / \mathrm{ARB}$ & $6030(43.43 \%)$ & 417 (53.12\%) & $<0.001$ & 727 (52.45\%) & 358 (51.66\%) & 0.733 \\
\hline$\alpha$-blocker & $1393(10.03 \%)$ & $90(11.46 \%)$ & 0.195 & $158(11.4 \%)$ & $79(11.4 \%)$ & 1.000 \\
\hline$\beta$-blocker & $4828(34.77 \%)$ & $325(41.4 \%)$ & $<0.001$ & $546(39.39 \%)$ & $278(40.12 \%)$ & 0.751 \\
\hline \multicolumn{7}{|l|}{ Calcium channel blocker } \\
\hline Non-DHP & $1439(10.36 \%)$ & 118 (15.03\%) & $<0.001$ & $213(15.37 \%)$ & $105(15.15 \%)$ & 0.897 \\
\hline DHP & $5544(39.93 \%)$ & $349(44.46 \%)$ & 0.012 & $628(45.31 \%)$ & $311(44.88 \%)$ & 0.852 \\
\hline \multicolumn{7}{|l|}{ Other Diuretics } \\
\hline Thiazide & $2298(16.55 \%)$ & $181(23.06 \%)$ & $<0.001$ & 313 (22.58\%) & 147 (21.21\%) & 0.478 \\
\hline Loop diuretics & $1623(11.69 \%)$ & 246 (31.34\%) & $<0.001$ & $343(24.75 \%)$ & $179(25.83 \%)$ & 0.592 \\
\hline Miscellaneous & $670(4.83 \%)$ & $62(7.9 \%)$ & $<0.001$ & $106(7.65 \%)$ & $50(7.22 \%)$ & 0.724 \\
\hline
\end{tabular}


Table 1. Cont.

\begin{tabular}{|c|c|c|c|c|c|c|}
\hline & \multicolumn{3}{|c|}{ Before Propensity-Score Matching } & \multicolumn{3}{|c|}{ After Propensity-Score Matching } \\
\hline & Non-User & User & $p$-Value & Non-User & User & $p$-Value \\
\hline \multicolumn{7}{|l|}{ Antidiabetic medication } \\
\hline Sulfonylurea & $3943(28.4 \%)$ & $277(35.29 \%)$ & $<0.001$ & $479(34.56 \%)$ & $241(34.78 \%)$ & 0.922 \\
\hline Meglitinide & $685(4.93 \%)$ & $51(6.5 \%)$ & 0.051 & $88(6.35 \%)$ & $41(5.92 \%)$ & 0.700 \\
\hline$\alpha$-glucosidase inhibitor & $998(7.19 \%)$ & $79(10.06 \%)$ & 0.003 & $147(10.61 \%)$ & $70(10.1 \%)$ & 0.723 \\
\hline Biguanide & $3611(26.01 \%)$ & $249(31.72 \%)$ & $<0.001$ & $437(31.53 \%)$ & $221(31.89 \%)$ & 0.868 \\
\hline Thiazolidinedione & $1013(7.3 \%)$ & $70(8.92 \%)$ & 0.091 & $111(8.01 \%)$ & $63(9.09 \%)$ & 0.401 \\
\hline Insulin & $937(6.75 \%)$ & $87(11.08 \%)$ & $<0.001$ & $146(10.53 \%)$ & $69(9.96 \%)$ & 0.684 \\
\hline Statins & $3724(26.82 \%)$ & $245(31.21 \%)$ & 0.007 & $430(31.02 \%)$ & $216(31.17 \%)$ & 0.947 \\
\hline Aspirin & $3753(27.03 \%)$ & $274(34.9 \%)$ & $<0.001$ & $492(35.5 \%)$ & $236(34.05 \%)$ & 0.516 \\
\hline NSAIDs & $2095(15.09 \%)$ & $149(18.98 \%)$ & 0.003 & $257(18.54 \%)$ & $126(18.18 \%)$ & 0.841 \\
\hline $\mathrm{NaHCO} 3$ & $147(1.06 \%)$ & $10(1.27 \%)$ & 0.569 & $13(0.94 \%)$ & $8(1.15 \%)$ & 0.642 \\
\hline Nephrology visit within 1 year before the index date & $1.4 \pm 2.1$ & $1.3 \pm 2.1$ & 0.243 & $1.3 \pm 2$ & $1.3 \pm 2.1$ & 0.994 \\
\hline Propensity score & $0.05 \pm 0.06$ & $0.14 \pm 0.17$ & $<0.001$ & $0.1 \pm 0.11$ & $0.1 \pm 0.11$ & 0.999 \\
\hline \multicolumn{7}{|l|}{ Outcome } \\
\hline ESRD & $2399(17.28 \%)$ & $102(12.99 \%)$ & 0.002 & $266(19.19 \%)$ & $88(12.7 \%)$ & $<0.001$ \\
\hline MACEs & $1084(7.81 \%)$ & $63(8.03 \%)$ & 0.878 & $123(8.87 \%)$ & $56(8.08 \%)$ & 0.599 \\
\hline Hospitalization for heart-failure & $423(3.05 \%)$ & $36(4.59 \%)$ & 0.021 & $76(5.48 \%)$ & $29(4.18 \%)$ & 0.243 \\
\hline Hyperkalemia-associated hospitalization & $724(5.21 \%)$ & $151(19.24 \%)$ & $<0.001$ & $92(6.64 \%)$ & $123(17.75 \%)$ & $<0.001$ \\
\hline Mortality & $2857(20.58 \%)$ & $226(28.79 \%)$ & $<0.001$ & $386(27.85 \%)$ & $192(27.71 \%)$ & 0.9448 \\
\hline CVD death & $413(3.0 \%)$ & $39(5.0 \%)$ & 0.002 & $70(5.1 \%)$ & $34(4.9 \%)$ & 0.972 \\
\hline
\end{tabular}

Abbreviations: PAOD, peripheral arterial occlusion disease; COPD, chronic obstructive pulmonary disease; CHF, congestive heart failure; ACEI, angiotensin-converting enzyme inhibitor; ARB, angiotensin II receptor blocker; DHP, dihydropyridine; NSAID, Non-Steroidal Anti-Inflammatory Drug; ESRD, end-stage renal disease; MACE, major adverse cardiovascular events; CVD, cardiovascular disease. 
Table 2. Risks for ESRD, MACEs, hospitalization for heart-failure, hyperkalemia-associated hospitalization and mortality among patients with stage 3-4 CKD by spironolactone use.

\begin{tabular}{|c|c|c|c|c|c|c|c|c|}
\hline & \multicolumn{2}{|r|}{ Users } & \multicolumn{2}{|c|}{ Non-Users } & \multicolumn{4}{|c|}{ Users Compared to Non-Users } \\
\hline & Event & IR $(95 \% \mathrm{CI})$ & Event & IR $(95 \%$ CI $)$ & Crude HR $(95 \% \mathrm{CI})$ & $p$-Value & Adjusted $\mathrm{HR}^{+}(95 \% \mathrm{CI})$ & $p$-Value \\
\hline ESRD & 88 & $39.2(31.01-47.39)$ & 266 & $53.69(47.24-60.14)$ & $0.65(0.51-0.83)$ & $<0.001$ & $0.66(0.51-0.84)$ & $<0.001$ \\
\hline MACE $\S$ & 56 & $24.94(18.41-31.48)$ & 123 & $24.83(20.44-29.21)$ & $0.93(0.68-1.27)$ & 0.647 & $0.93(0.67-1.28)$ & 0.647 \\
\hline Hospitalization for heart-failure & 29 & $12.92(8.22-17.62)$ & 76 & $15.34(11.89-18.79)$ & $0.77(0.50-1.19)$ & 0.238 & $0.77(0.50-1.18)$ & 0.225 \\
\hline Hyperkalemia-associated hospitalization & 123 & $54.79(45.1-64.47)$ & 92 & $18.57(14.77-22.36)$ & $2.98(2.28-3.90)$ & $<0.001$ & $3.17(2.41-4.17)$ & $<0.001$ \\
\hline All-cause mortality & 192 & $64.42(55.31-73.53)$ & 386 & $60.47(54.44-66.5)$ & $1.07(0.90-1.27)$ & 0.432 & $1.10(0.92-1.30)$ & 0.294 \\
\hline Cardiovascular death & 34 & $11.41(7.57-15.24)$ & 70 & $10.97(8.4-13.53)$ & $1.02(0.67-1.53)$ & 0.941 & $1.14(0.75-1.74)$ & 0.533 \\
\hline
\end{tabular}

Abbreviation: CI, confidence interval; HR, hazard ratio; IR, incidence rate (per 1000 person-years). ESRD, end-stage renal disease; MACE, major adverse cardiovascular events. ${ }^{\dagger}$ Adjusted

for all covariates in Table 1 after propensity-score matching. $\$$ MACE, the composite of acute myocardial infarction and ischemic stroke. 
Table 3. Risks for ESRD, MACEs, HHF, HKAH and mortality among patients with stage 3-4 CKD by prescribed daily dose and cumulative defined daily dose of spironolactone within 90 days.

\begin{tabular}{|c|c|c|c|c|c|c|c|c|c|c|c|c|}
\hline & \multicolumn{12}{|c|}{ Outcomes } \\
\hline & \multicolumn{2}{|c|}{ ESRD } & \multicolumn{2}{|c|}{ MACE § } & \multicolumn{2}{|c|}{ HHF } & \multicolumn{2}{|c|}{ НКАН } & \multicolumn{2}{|c|}{ All-Cause Mortality } & \multicolumn{2}{|c|}{ CVD Mortality } \\
\hline & $\begin{array}{l}\text { Adj. HR }{ }^{\dagger} \\
(95 \% \mathrm{CI})\end{array}$ & $p$-Value & $\begin{array}{l}\text { Adj. HR }{ }^{\dagger} \\
(95 \% \text { CI })\end{array}$ & $p$-Value & $\begin{array}{l}\text { Adj. HR }{ }^{\dagger} \\
(95 \% \mathrm{CI})\end{array}$ & $p$-Value & $\begin{array}{l}\text { Adj. HR }{ }^{\dagger} \\
(95 \% \text { CI })\end{array}$ & $p$-Value & $\begin{array}{c}\text { Adj. HR }{ }^{\dagger} \\
(95 \% \text { CI })\end{array}$ & $p$-Value & $\begin{array}{c}\text { Adj. HR }{ }^{\dagger} \\
(95 \% \text { CI })\end{array}$ & $p$-Value \\
\hline \multicolumn{13}{|c|}{ Prescribed daily dose (mg) } \\
\hline \multicolumn{13}{|c|}{ Spironolactone (vs. non-use) } \\
\hline$<12.5 \mathrm{mg}$ & $\begin{array}{c}0.73 \\
(0.52-1.00)\end{array}$ & 0.0523 & $\begin{array}{c}0.97 \\
(0.64-1.45)\end{array}$ & 0.8688 & $\begin{array}{c}0.78 \\
(0.43-1.41)\end{array}$ & 0.4102 & $\begin{array}{c}2.81 \\
(1.96-4.03)\end{array}$ & $<0.0001$ & $\begin{array}{c}1.15 \\
(0.91-1.45)\end{array}$ & 0.2304 & $\begin{array}{c}0.97 \\
(0.54-1.71)\end{array}$ & 0.904 \\
\hline $12.5-25 \mathrm{mg}$ & $\begin{array}{c}0.59 \\
(0.39-0.91)\end{array}$ & 0.0175 & $\begin{array}{c}0.57 \\
(0.29-1.11)\end{array}$ & 0.0965 & $\begin{array}{c}0.90 \\
(0.45-1.81)\end{array}$ & 0.7732 & $\begin{array}{c}2.74 \\
(1.8-4.17)\end{array}$ & $<0.0001$ & $\begin{array}{c}1.15 \\
(0.87-1.53)\end{array}$ & 0.3302 & $\begin{array}{c}1.34 \\
(0.73-2.46)\end{array}$ & 0.3437 \\
\hline$\geq 25 \mathrm{mg}$ & $\begin{array}{c}0.57 \\
(0.35-0.91)\end{array}$ & 0.0193 & $\begin{array}{c}1.34 \\
(0.79-2.25)\end{array}$ & 0.2803 & $\begin{array}{c}0.61 \\
(0.26-1.44)\end{array}$ & 0.2572 & $\begin{array}{c}4.80 \\
(3.3-6.97)\end{array}$ & $<0.0001$ & $\begin{array}{c}0.95 \\
(0.70-1.29)\end{array}$ & 0.744 & $\begin{array}{c}0.87 \\
(0.41-1.84)\end{array}$ & 0.7107 \\
\hline$p$-trend & & 0.0057 & & 0.2428 & & 0.5971 & & $<0.0001$ & & 0.4920 & & 0.7568 \\
\hline \multicolumn{13}{|c|}{ Cumulative defined daily dose (cDDD) (vs. non-use) } \\
\hline \multicolumn{13}{|l|}{ Spironolactone } \\
\hline$\leq 30 \mathrm{cDDD}$ & $\begin{array}{c}0.68 \\
(0.52-0.89)\end{array}$ & 0.005 & $\begin{array}{c}0.82 \\
(0.57-1.19)\end{array}$ & 0.2951 & $\begin{array}{c}0.84 \\
(0.53-1.34)\end{array}$ & 0.4749 & $\begin{array}{c}2.77 \\
(2.04-3.75)\end{array}$ & $<0.0001$ & $\begin{array}{c}1.16 \\
(0.96-1.4)\end{array}$ & 0.1312 & $\begin{array}{c}1.09 \\
(0.69-1.7)\end{array}$ & 0.7206 \\
\hline$>30 \mathrm{cDDD}$ & $\begin{array}{c}0.60 \\
(0.37-0.97)\end{array}$ & 0.0353 & $\begin{array}{c}1.36 \\
(0.80-2.33)\end{array}$ & 0.2600 & $\begin{array}{c}0.53 \\
(0.20-1.37)\end{array}$ & 0.1903 & $\begin{array}{c}4.70 \\
(3.21-6.87)\end{array}$ & $<0.0001$ & $\begin{array}{c}0.94 \\
(0.68-1.28)\end{array}$ & 0.6708 & $\begin{array}{c}0.89 \\
(0.42-1.9)\end{array}$ & 0.7695 \\
\hline$p$-trend & & 0.0038 & & 0.2588 & & 0.3522 & & $<0.0001$ & & 0.2535 & & 0.9644 \\
\hline
\end{tabular}




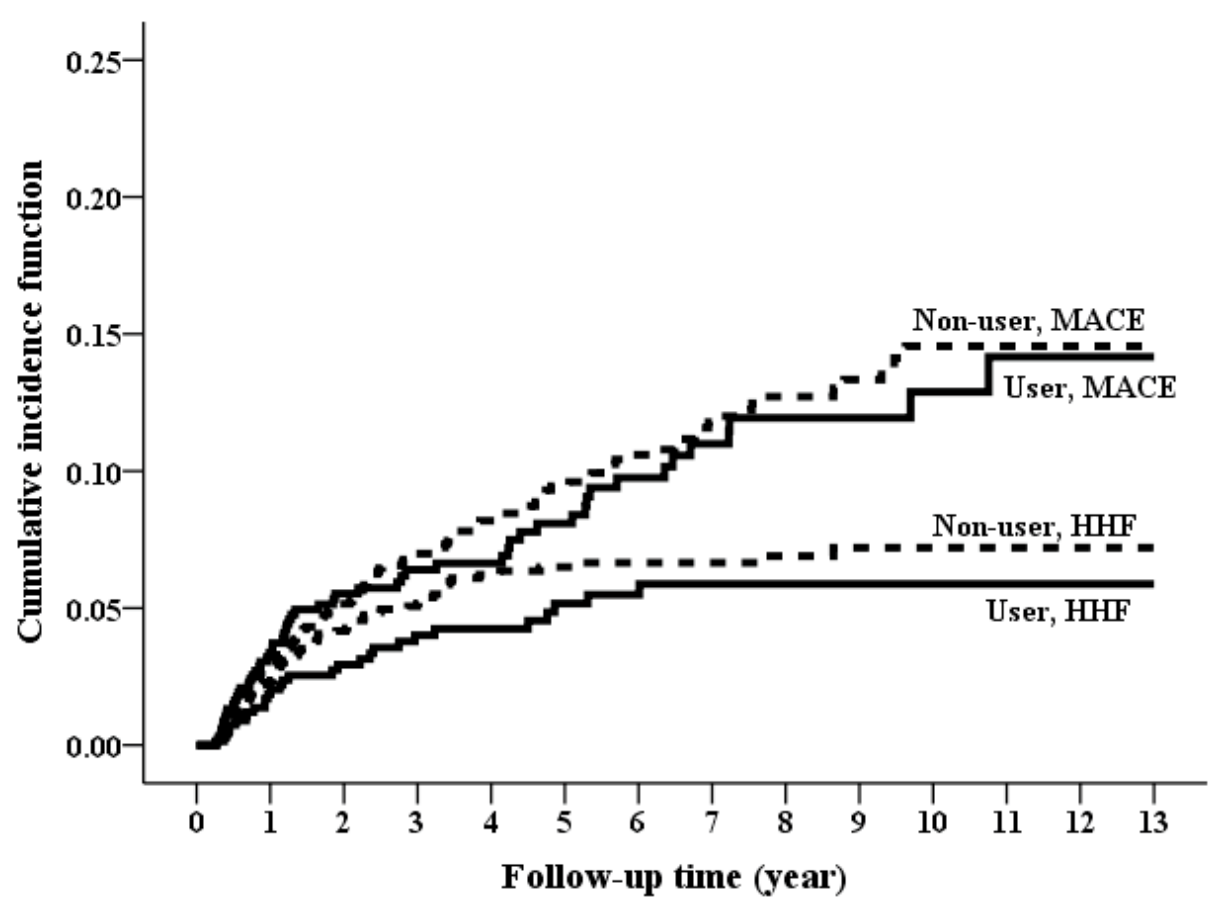

P-value $=0.7052$ by Gray's test for MACE

$P$-value $=0.2554$ by Gray's test for HHF

Figure 4. Cumulative incidence rate of hospitalization for heart failure ( $p$-value $=0.2554$, Grey's test) and major adverse cardiovascular events ( $p$-value $=0.7052$, Grey's test) between spironolactone users and non-users between spironolactone users and non-users.

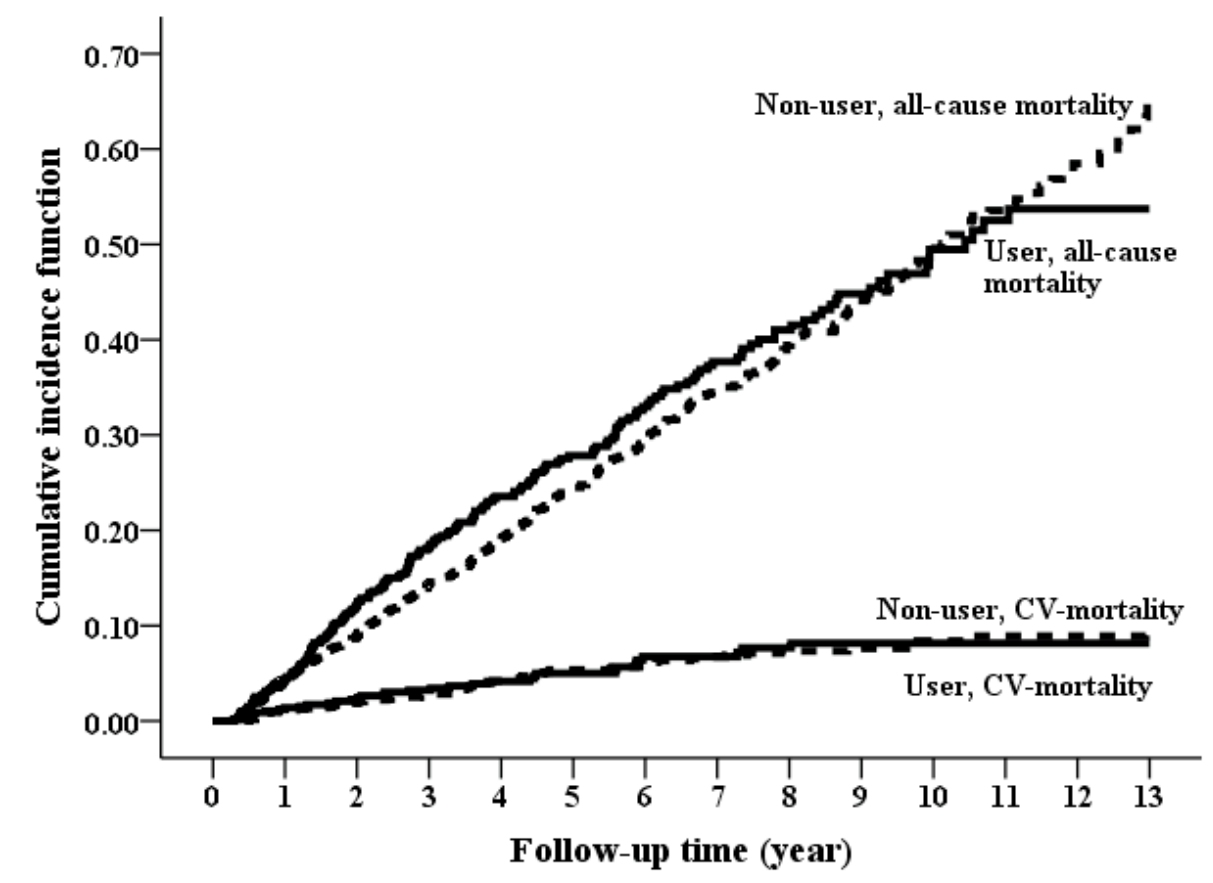

P-value $=0.4390$ by Gray's test for all-cause mortality P-value $=0.9408$ by Gray's test for CV-mortality

Figure 5. Cumulative incidence rate of all-cause mortality ( $p$-value $=0.439$, Grey's test) and cardiovascular disease-mortality ( $p$-value $=0.9408$, Grey's test) between spironolactone users and non-users. 


\subsection{Subgroup Analysis}

The reduced HRs of ESRD associated with spironolactone use among moderate to severe CKD patients were consistent across most of the patient subgroups, except those with female cohort, no nephrological visit, non-hypertension, DM, stroke, cirrhosis, ACEI/ARB users, while the significant interaction effect was only found between ESRD and DM (Figure 6). Moreover, the increased HRs of HKAH associated with spironolactone use were consistently significant in all the stratified subgroups (Figure 7).

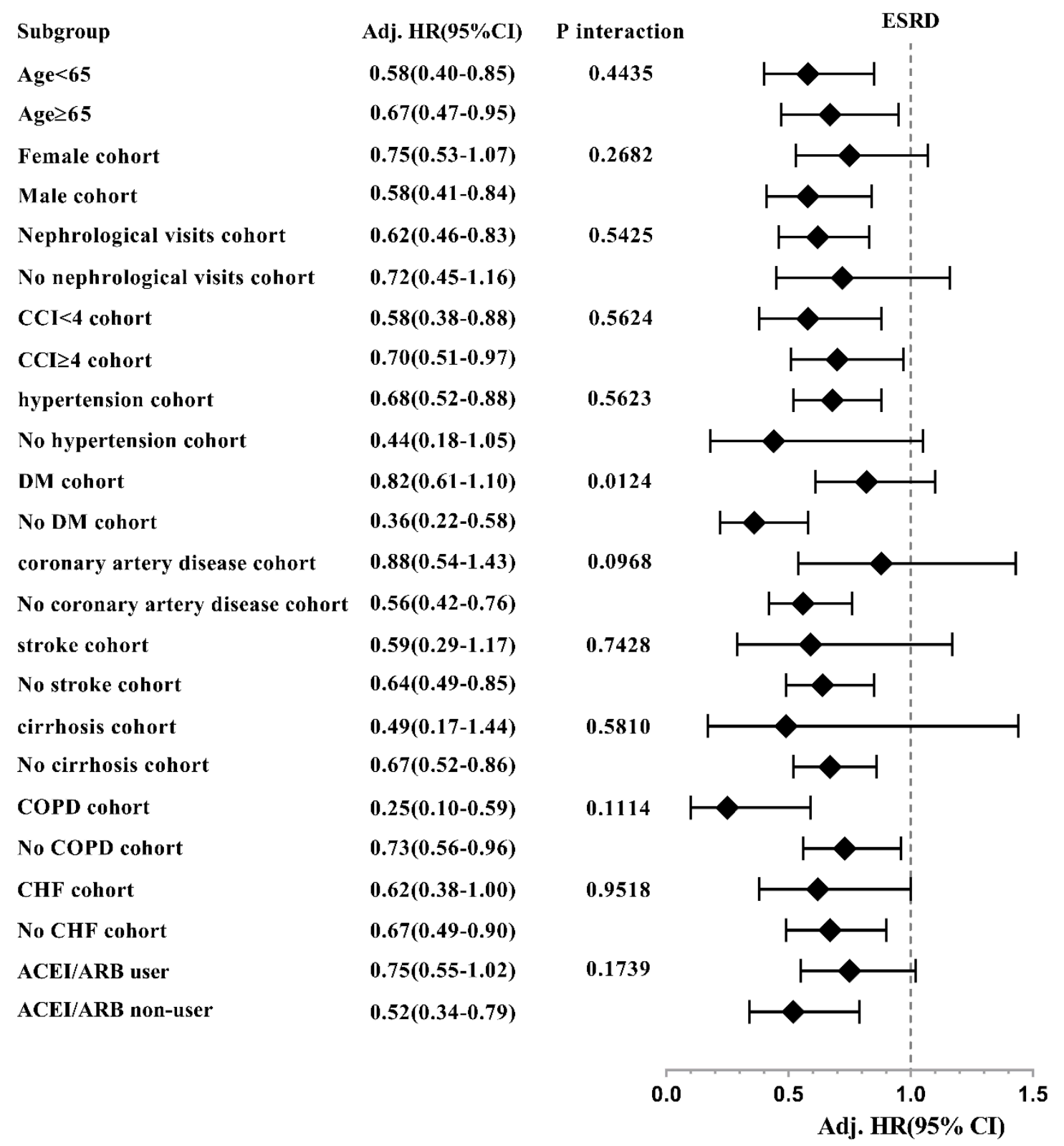

Figure 6. Hazard ratios of end-stage renal disease associated with spironolactone use in subgroup analyses. 


\begin{tabular}{|c|c|c|c|}
\hline Subgroup & Adj. HR(95\%CI) & $P$ interaction & $\begin{array}{c}\text { Incident of hyperkalemia-associated } \\
\text { hospitalization }\end{array}$ \\
\hline Age $<65$ & $6.06(3.69-9.96)$ & 0.0043 & -1 \\
\hline Age $\geq 65$ & $2.37(1.66-3.38)$ & & \\
\hline Female cohort & $3.55(2.33-5.41)$ & 0.6547 & \\
\hline Male cohort & $3.17(2.17-4.61)$ & & \\
\hline Nephrological visits cohort & $3.32(2.32-4.75)$ & 0.8578 & \\
\hline No nephrological visits cohort & $3.06(1.99-4.70)$ & & \\
\hline CCI $<4$ cohort & $5.00(3.06-8.17)$ & 0.0597 & ! \\
\hline $\mathrm{CCI} \geq 4$ cohort & $2.58(1.84-3.62)$ & & \\
\hline hypertension cohort & $2.97(2.16-4.09)$ & 0.1981 & ! \\
\hline No hypertension cohort & $5.21(2.54-10.66)$ & & \\
\hline DM cohort & $3.31(2.27-4.82)$ & 0.7877 & \\
\hline No DM cohort & $3.25(2.12-4.99)$ & & \\
\hline coronary artery disease cohort & $2.87(1.72-4.81)$ & 0.4694 & \\
\hline No coronary artery disease cohort & $3.58(2.56-5.01)$ & & \\
\hline stroke cohort & $2.73(1.30-5.71)$ & 0.6866 & \\
\hline No stroke cohort & $3.23(2.39-4.38)$ & & \\
\hline cirrhosis cohort & $7.11(1.92-26.30)$ & 0.7315 & 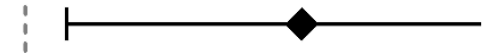 \\
\hline No cirrhosis cohort & $3.29(2.44-4.44)$ & & \\
\hline COPD cohort & $2.01(1.07-3.78)$ & 0.1113 & \\
\hline No COPD cohort & $3.59(2.60-4.96)$ & & i \\
\hline CHF cohort & $2.93(1.74-4.95)$ & 0.4792 & \\
\hline No CHF cohort & $3.46(2.47-4.86)$ & & \\
\hline ACEI/ARB user & $3.00(2.05-4.39)$ & 0.5369 & 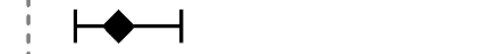 \\
\hline \multirow[t]{2}{*}{ ACEI/ARB non-user } & $3.94(2.48-6.28)$ & & i \\
\hline & & 0.0 & 10.0 \\
\hline
\end{tabular}

Figure 7. Hazard ratios of progression to hyperkalemia-associated hospitalization associated with spironolactone use in subgroup analyses.

\subsection{Sensitivity Analyses}

The results of a series of sensitivity analyses were shown in Table 4. All the sensitivity tests revealed a lower risk of ESRD and a higher risk of HKAH associated with spironolactone use, indicating the robustness of our findings. 
Table 4. Sensitivity Analyses.

\begin{tabular}{|c|c|c|c|c|c|c|c|c|c|c|c|c|}
\hline & \multicolumn{12}{|c|}{ Outcomes } \\
\hline & \multicolumn{2}{|c|}{ ESRD } & \multicolumn{2}{|c|}{ MACE $\S$} & \multicolumn{2}{|c|}{ HHF } & \multicolumn{2}{|c|}{ HKAH } & \multicolumn{2}{|c|}{ All-Cause Mortality } & \multicolumn{2}{|c|}{ CVD-Mortality } \\
\hline & $\begin{array}{c}\text { Adj. HR }{ }^{\dagger} \\
(95 \% \text { CI })\end{array}$ & $p$-Value & $\begin{array}{c}\text { Adj. HR }{ }^{\dagger} \\
(95 \% \text { CI })\end{array}$ & $p$-Value & $\begin{array}{c}\text { Adj. HR }{ }^{\dagger} \\
(95 \% \text { CI })\end{array}$ & $p$-Value & $\begin{array}{l}\text { Adj. HR }{ }^{\dagger} \\
(95 \% \text { CI })\end{array}$ & $p$-Value & $\begin{array}{c}\text { Adj. HR }{ }^{\dagger} \\
(95 \% \text { CI })\end{array}$ & $p$-Value & $\begin{array}{c}\text { Adj. HR }{ }^{\dagger} \\
(95 \% \text { CI })\end{array}$ & $p$-Value \\
\hline \multicolumn{13}{|c|}{ Time intervals for defining Spironolactone users/non-users } \\
\hline \multicolumn{13}{|l|}{ Within 60 days } \\
\hline Spironolactone use (vs. non-use) & $\begin{array}{c}0.59 \\
(0.46-0.77)\end{array}$ & $<0.0001$ & $\begin{array}{c}1.08 \\
(0.77-1.50)\end{array}$ & 0.6618 & $\begin{array}{c}0.82 \\
(0.53-1.28)\end{array}$ & 0.3861 & $\begin{array}{c}2.40 \\
(1.82-3.16)\end{array}$ & $<0.0001$ & $\begin{array}{c}1.09 \\
(0.91-1.30)\end{array}$ & 0.3495 & $\begin{array}{c}1.20 \\
(0.80-1.82)\end{array}$ & 0.3785 \\
\hline \multicolumn{13}{|l|}{ Within 120 days } \\
\hline Spironolactone use (vs. non-use) & $\begin{array}{c}0.73 \\
(0.58-0.92)\end{array}$ & 0.0088 & $\begin{array}{c}0.96 \\
(0.70-1.32)\end{array}$ & 0.8003 & $\begin{array}{c}1.12 \\
(0.74-1.71)\end{array}$ & 0.5816 & $\begin{array}{c}2.52 \\
(1.94-3.26)\end{array}$ & $<0.0001$ & $\begin{array}{c}1.01 \\
(0.85-1.19)\end{array}$ & 0.9148 & $\begin{array}{c}1.12 \\
(0.18-7.09)\end{array}$ & 0.904 \\
\hline \multicolumn{13}{|l|}{ Within 180 days } \\
\hline Spironolactone use (vs. non-use) & $\begin{array}{c}0.69 \\
(0.55-0.87)\end{array}$ & 0.0019 & $\begin{array}{c}0.95 \\
(0.68-1.32)\end{array}$ & 0.7508 & $\begin{array}{c}0.84 \\
(0.54-1.3)\end{array}$ & 0.4367 & $\begin{array}{c}3.08 \\
(2.28-4.16)\end{array}$ & $<0.0001$ & $\begin{array}{c}1.01 \\
(0.85-1.21)\end{array}$ & 0.8976 & $\begin{array}{c}1.00 \\
(0.62-1.61)\end{array}$ & 0.9847 \\
\hline \multicolumn{13}{|c|}{ Exclude control cohort receiving spironolactone during the follow-up period } \\
\hline Spironolactone use (vs. non-use) & $\begin{array}{c}0.63 \\
(0.49-0.81)\end{array}$ & 0.0002 & $\begin{array}{c}0.91 \\
(0.66-1.26)\end{array}$ & 0.5724 & $\begin{array}{c}0.76 \\
(0.49-1.19)\end{array}$ & 0.2263 & $\begin{array}{c}3.65 \\
(2.71-4.91)\end{array}$ & $<0.0001$ & $\begin{array}{c}1.07 \\
(0.89-1.27)\end{array}$ & 0.4773 & $\begin{array}{c}1.11 \\
(0.70-1.78)\end{array}$ & 0.6565 \\
\hline \multicolumn{13}{|c|}{ Consider spironolactone status change as censored (As-treat model) } \\
\hline Spironolactone use (vs. non-use) & $\begin{array}{c}0.37 \\
(0.21-0.64)\end{array}$ & 0.0004 & $\begin{array}{c}0.71 \\
(0.44-1.14)\end{array}$ & 0.1553 & $\begin{array}{c}1.11 \\
(0.65-1.92)\end{array}$ & 0.6967 & $\begin{array}{c}9.18 \\
(6.69-12.6)\end{array}$ & $<0.0001$ & $\begin{array}{c}1.17 \\
(0.83-1.66)\end{array}$ & 0.3642 & $\begin{array}{c}1.42 \\
(0.68-2.97)\end{array}$ & 0.3571 \\
\hline \multicolumn{13}{|l|}{ Cohort in 2000-2006 } \\
\hline Spironolactone use (vs. non-use) & $\begin{array}{c}0.48 \\
(0.28-0.81)\end{array}$ & 0.0064 & $\begin{array}{c}1.17 \\
(0.60-2.28)\end{array}$ & 0.6515 & $\begin{array}{c}1.02 \\
(0.24-4.27)\end{array}$ & 0.9828 & $\begin{array}{c}2.38 \\
(1.3-4.35)\end{array}$ & 0.0048 & $\begin{array}{c}1.34 \\
(0.76-2.37)\end{array}$ & 0.3125 & $\begin{array}{c}1.34 \\
(0.35-5.07)\end{array}$ & 0.6672 \\
\hline \multicolumn{13}{|l|}{ Cohort in 2007-2013 } \\
\hline Spironolactone use (vs. non-use) & $\begin{array}{c}0.71 \\
(0.53-0.94)\end{array}$ & 0.0184 & $\begin{array}{c}0.88 \\
(0.61-1.27)\end{array}$ & 0.5035 & $\begin{array}{c}0.73 \\
(0.43-1.21)\end{array}$ & 0.2167 & $\begin{array}{c}3.33 \\
(2.45-4.52)\end{array}$ & $<0.0001$ & $\begin{array}{c}1.18 \\
(0.98-1.43)\end{array}$ & 0.0818 & $\begin{array}{c}1.16 \\
(0.74-1.82)\end{array}$ & 0.5137 \\
\hline \multicolumn{13}{|c|}{ Using raw data (before propensity-score matching) as analyzed data } \\
\hline Spironolactone use (vs. non-use) & $\begin{array}{c}0.65 \\
(0.53-0.80)\end{array}$ & $<0.0001$ & $\begin{array}{c}1.02 \\
(0.78-1.33)\end{array}$ & 0.9091 & $\begin{array}{c}0.85 \\
(0.59-1.21)\end{array}$ & 0.3649 & $\begin{array}{c}3.00 \\
(2.46-3.67)\end{array}$ & $<0.0001$ & $\begin{array}{c}1.21 \\
(1.04-1.40)\end{array}$ & 0.0141 & $\begin{array}{c}1.23 \\
(0.27-5.62)\end{array}$ & 0.7899 \\
\hline
\end{tabular}

Abbreviation: Adj. HR = Adjusted Hazard Ratio; CI, confidence interval; ESRD, end-stage renal disease; MACE, major adverse cardiovascular events; HHF, hospitalization for heart-failure, $\mathrm{HKAH}$, hyperkalemia-associated hospitalization. ${ }^{\dagger}$ Adjusted for all covariates in Table 1 after propensity-score matching. ${ }^{\S} \mathrm{MACE}$, the composite of acute myocardial infarction and ischemic stroke. 


\section{Discussion}

As far as we know, the present study was the first and largest study of nationwide cohort population to assess the effects of spironolactone on patient-centered hard endpoints in patients with moderate to severe (stage 3-4) CKD. The most striking finding was, for the first time, that treatment with spironolactone was associated with a lower risk of ESRD but complicated with a higher risk of HKAH. The dose-dependent effect of spironolactone suggested that a lower risk of ESRD was confounded by a higher risk of HKAH while prescribing higher doses of spironolactone. Insignificant associations were found between spironolactone users and non-users in terms of MACE, HHF, all-cause mortality, and cardiovascular mortality. The associations were further consolidated by the consistent results throughout the sensitivity tests and in most subgroups of patients.

A growing body of clinical evidence has been emerging in support of the independent role of aldosterone in the development and progression in cardiovascular and kidney disease [11-13]. The majority of the studies on spironolactone among CKD patients focused on the change in urinary protein/albumin secretion, blood pressure, serum potassium levels and creatinine clearance or estimated glomerular filtration rate in the presence of ACEI and/or ARB treatment [14-17]. The study duration ranged from 8 to 52 weeks. The patient population was small $(n=18-208)$ with variable methodology and most of them had stage 1-3 CKD. Data were not available on long-term patient-focused outcomes, including cardiovascular events, ESRD, and mortality in any of the trials. Currie et al. demonstrated that combined use of MRA with ACEI and/or ARB significantly lowered proteinuria with a higher risk of hyperkalemia, but was associated with a small, non-significant decline in renal function in a recent meta-analysis [18]. An average increase of $0.19 \mathrm{mmol} / \mathrm{L}$ in serum potassium level and three-fold higher risk of hyperkalemia were shown when CKD patients received MRA in addition to ACEI and/or ARB. Our findings were partially in line with those previous studies and extended to clinical endpoints. By using a representative nationwide cohort data with an appropriate follow-up time and 2-to-1 propensity-score matching, we are confident with the reduced risk of ESRD and higher risk of HKAH for spironolactone use in moderate to severe CKD patients. Spironolactone was associated with 34\% reduced risk of ESRD and a three times greater risk of HKAH. Therefore, the renoprotective benefit of spironolactone however may be offset by the hyperkalemia risk.

The hyperkalemia risk induced by spironolactone may be mitigated by replacement with nonsteroidal MRA which reportedly had a promising reduction in albuminuria with a lower risk of side effects in patients with diabetic nephropathy [19]. Furthermore, the concomitant use of potassium-lowering agent when prescribing spironolactone and/or other RAAS inhibitors for high-risk patients may also decrease hyperkalemia occurrence. The economic impact of more frequent monitoring of serum potassium concentrations should be evaluated against the beneficial reduction of CKD progression.

Mineralocorticoid receptors (MR) are expressed in podocytes, mesangial cells and endothelial cells and smooth muscle cells of murine vasculature [20]. Renal MR were upregulated in animals models of both type 1 and type 2 DM [21]. In kidney biopsies of patients with renal failure, renal MR mRNA expression in those with heavy albuminuria is 4.6 times higher than those with no albuminuria, microalbuminuria and moderate albuminuria [22]. Aldosterone-mediated renal injury is mainly ascribed to inflammation and fibrosis, independent of the systemic effects on blood pressure. The deleterious effects of aldosterone on the kidneys include glomerular hypertrophy, glomerulosclerosis, proteinuria, and reduced renal blood flow through the upregulation of NADPH oxidase activity, reactive oxygen species, nuclear factor $-\mathrm{k} \beta$, pro-inflammatory cytokines and pro-fibrotic proteins [23]. The most convincing evidence for the detrimental role of MR may be derived from the demonstration of MRA, spironolactone or eplerenone, to have renoprotective effects in animal models of kidney diseases. MRA was shown to suppress MR expression, and reduce makers of oxidative stress, pro-inflammatory and pro-fibrotic mediators, including TGF- $\beta$, connective tissue growth factor, and osteopontin, in murine models [20,24-27]. MRA can also attenuate apoptosis and endothelial dysfunction with increases in endothelial nitric oxide synthase [28-30]. Further benefits 
of MRA include improvement of podocyte injury, glomerulosclerosis, proteinuria and glomerular hypertrophy [31-33]. Taken together, the reduced risk of ESRD by spironolactone may be ascribed to jointly those favorable hemodynamic changes and metabolic effects of spironolactone.

Recently, Tseng et al. reported the use of spironolactone in advanced (stage 5) CKD patients resulted in higher risks of all-cause mortality, HHF, and infection related mortality and non-significant associations with MACE, CVD mortality and HKAH whereas the impact on ESRD was not evaluated [34]. They attributed the higher mortality risk to the exacerbation of the already present metabolic acidosis, hyperuricemia, endocrinopathy (cortisol, testosterone and glucose homeostasis) and impairment of host immunity after the use of spironolactone. The distinct results from ours may be that our cohort comprised stage 3-4 CKD patients who were not so susceptible to the deleterious metabolic effects by spironolactone. Notably, our intriguing finding of HKAH risk was not present in their stage 5 CKD patients. Potassium balance is usually maintained until reaching stage 5 CKD where more than one-half of patients had mild elevation of potassium. In their study, not only the spironolactone use group but also the non-use group had a higher incidence of HKAH than our groups of spironolactone use and non-use, suggesting that basically stage 5 CKD patients without taking spironolactone even had a higher HKAH risk than stage 3-4 CKD patients with spironolactone treatment. The crude HR of HKAH was not significant between the spironolactone users and non-users in stage 5 CKD patients, whereas a significantly higher risk was seen in stage 3-4 CKD patients treated with spironolactone. Therefore, the effect of spironolactone-induced HKAH was pronounced only in stage 3-4 CKD patients whose HKAH was extremely low without spironolactone use, but not in stage 5 CKD patients who had pre-existing relatively right HKAH even in the absence of spironolactone treatment.

The present study is the first large-scale nationwide one to investigate the effects of spironolactone on several clinical endpoints in stage 3-4 CKD patients. The selection bias was minimized due to the use of medical claim data of NHIRD, which covered more than $99 \%$ of Taiwanese residents. In addition, a longer follow-up time and more enrolled patients than previously published trials allowed us to assess the risks and benefits associated with spironolactone in the long run. Furthermore, the robustness of our findings was strengthened by performing propensity-score matching process, sensitivity analyses and subgroup tests. However, some limitations should also be addressed. First, some residual confounding factors may affect our outcomes despite we managed to adjust for clinically important patient characteristics, including socio-economic status, relevant comorbidities and medications usage. The NHIRD also did not contain personal information, which is known as key determinants of clinical outcomes, such as medical adherence, over-the-counter medications, Chinese herbal medicine, tobacco use, and laboratory measurements. A propensity-score matching method was applied to control for the residual confounders. The unmeasured confounders were likely to distribute equally in the spironolactone and control groups. Second, causality cannot be approved in our retrospective observational study. Our national cohort study represented a clinical practice in the real world, unlike the randomized controlled trials where women and high-risk and vulnerable populations are usually excluded. Available evidence also indicated that well-designed observational studies do not systemically overestimate the treatment effects and can yield comparable results to randomized controlled trials [35].

Third, the use status of spironolactone may change over time, biasing the statistical analyses. We defined the spironolactone users by several time intervals and ran the analyses by treating spironolactone use in as-treated model and excluding the control cohort receiving spironolactone during the follow-up period, and checked the cohort effects. All of these tests produced similar results as the primary analyses. Fourthly, there is indication bias inherent to this study because spironolactone was not prescribed with the intention to prevent the progression of CKD. Instead, spironolactone is mainly used as treatment for cirrhosis, resistant hypertension or heart failure and these comorbidities increase the progression of CKD to ESRD. Therefore, the spironolactone users were more frequently co-morbid, especially by cirrhosis, resistant hypertension and heart failure, which was evident from our 
unmatched data for baseline characteristics. Because the spironolactone users were more comorbid and these comorbidities worsen renal function, the renal protective effect of spironolactone was supposed to augment in the absence of these comorbidities. We also re-ran the multivariate regression analysis using the raw data before propensity score matching and the results were consistent with the primary analyses. Finally, the results of the present study may not be applicable to other population outside Taiwan due to different ethnicity, cultural background and health service systems. It may only apply to stage 3-4 CKD patients, not to advanced (stage 5) CKD.

In conclusion, spironolactone represented a promising treatment option to retard CKD progression to ESRD in patients with moderate to severe (stage 3-4) CKD. The HR of ESRD for spironolactone users versus non-users was $0.66(0.51-0.84)$ but it also carried a significantly quantifiable risk of HKAH. There was no significant difference in the risks of MACE, HHF, all-cause mortality and CVD mortality between spironolactone users and non-users. Based on the results of our nationwide population-based cohort study, spironolactone can be prescribed for its renoprotective effect in stage 3-4 CKD but strategic treatments to prevent hyperkalemia should be enforced.

Author Contributions: Conceptualization, C.-T.Y.; Formal analysis, C.-T.K.; Writing—original draft, Y.-P.H.

Funding: This study was funded by grants 107-CCH-IRP-029 from the Changhua Christian Hospital Research Foundation.

Conflicts of Interest: There is no competing interest.

\section{References}

1. Gansevoort, R.T.; Correa-Rotter, R.; Hemmelgarn, B.R.; Jafar, T.H.; Heerspink, H.J.; Mann, J.F.; Matsushita, K.; Wen, C.P. Chronic kidney disease and cardiovascular risk: Epidemiology, mechanisms, and prevention. Lancet 2017, 382, 339-352. [CrossRef]

2. Tonelli, M.; Wiebe, N.; Culleton, B.; House, A.; Rabbat, C.; Fok, M.; McAlister, F.; Garg, A.X. Chronic kidney disease and mortality risk: A systematic review. J. Am. Soc. Nephrol. 2006, 17, 2034-2047. [CrossRef] [PubMed]

3. Tomiyama, C.; Higa, A.; Dalboni, M.A.; Cendoroglo, M.; Draibe, S.A.; Cuppari, L.; Carvalho, A.B.; Neto, E.M.; Canziani, M.E. The impact of traditional and nontraditional risk factors on coronary calcification in pre-dialysis patients. Nephrol. Dial. Transpl. 2006, 21, 2464-2471. [CrossRef] [PubMed]

4. Brenner, B.M.; Cooper, M.E.; de Zeeuw, D.; Keane, W.F.; Mitch, W.E.; Parving, H.H.; Remuzzi, G.; Snapinn, S.M.; Zhang, Z.; Shahinfar, S.; et al. Effects of losartan on renal and cardiovascular outcomes in patients with type 2 diabetes and nephropathy. N. Engl. J. Med. 2001, 345, 861-869. [CrossRef] [PubMed]

5. Jafar, T.H.; Schmid, C.H.; Landa, M.; Giatras, I.; Toto, R.; Remuzzi, G.; Maschio, G.; Brenner, B.M.; Kamper, A.; Zucchelli, P.; et al. Angiotensin-converting enzyme inhibitors and progression of nondiabetic renal disease. A meta-analysis of patient-level data. Ann. Intern. Med. 2001, 135, 73-87. [CrossRef] [PubMed]

6. Ma, L.J.; Fogo, A.B. Role of angiotensin II in glomerular injury. Semin. Nephrol. 2001, 21, 544-553. [CrossRef] [PubMed]

7. McElvie, R.S.; Yusuf, S.; Pericak, D.; Avezum, A.; Burns, R.J.; Probstfield, J.; Tsuyuki, R.T.; White, M.; Rouleau, J.; Latini, R.; et al. Comparison of candesartan, enalapril, and their combination in congestive heart failure: Randomized evaluation of strategies for left ventricular dysfunction (RESOLVD) pilot study. Circulation 1999, 10, 1056-1064. [CrossRef]

8. Bolignano, D.; Palmer, S.C.; Navaneethan, S.D.; Strippoli, G.F. Aldosterone antagonists for preventing the progression of chronic kidney disease. Cochrane Database Syst. Rev. 2014, 4, CD007004. [CrossRef] [PubMed]

9. Ng, K.P.; Arnold, J.; Sharif, A.; Gill, P.; Townend, J.N.; Ferro, C.J. Cardiovascular actions of mineralocorticoid receptor antagonists in patients with chronic kidney disease: A systematic review and meta-analysis. J. Renin. Angiotensin Aldosterone Syst. 2015, 16, 599-613. [CrossRef] [PubMed]

10. Epstein, M. Mineralocorticoid receptor antagonists: Part of an emerging treatment paradigm for chronic kidney disease. Lancet Diabetes Endocrinol. 2014, 2, 925-927. [CrossRef] 
11. Alfie, J.; Aparicio, L.S.; Waisman, G.D. Current strategies to achieve further cardiac and renal protection through enhanced renin-angiotensin-aldosterone system inhibition. Rev. Recent Clin. Trials 2011, 6, 134-146. [PubMed]

12. Schjoedt, K.J.; Andersen, S.; Rossing, P.; Tarnow, L.; Parving, H.H. Aldosterone escape during blockade of the renin-angiotensin-aldosterone system in diabetic nephropathy is associated with enhanced decline in glomerular filtration rate. Diabetologia 2004, 47, 1936-1939. [CrossRef] [PubMed]

13. Cerezo, C.; Ruilope, L.M.; Segura, J.; Garcia-Donaire, J.A.; de la Cruz, J.J.; Banegas, J.R.; Waeber, B.; Rabelink, T.J.; Messerli, F.H. Microalbuminuria breakthrough under chronic renin-angiotensin-aldosterone system suppression. J. Hypertens. 2012, 30, 204-209. [CrossRef] [PubMed]

14. Edwards, N.C.; Steeds, R.P.; Stewart, P.M.; Ferro, C.J.; Townend, J.N. Effect of spironolactone on left ventricular mass and aortic stiffness in early-stage chronic kidney disease: A randomized controlled trial. J. Am. Coll. Cardiol. 2009, 54, 505-512. [CrossRef] [PubMed]

15. Rossing, K.; Schjoedt, K.J.; Smidt, U.M.; Boomsma, F.; Parving, H.H. Beneficial effects of adding spironolactone to recommended antihypertensive treatment in diabetic nephropathy: A randomized, double-masked, cross-over study. Diabetes Care 2005, 28, 2106-2112. [CrossRef] [PubMed]

16. Tylicki, L.; Rutkowski, P.; Renke, M.; Larczyński, W.; Aleksandrowicz, E.; Lysiak-Szydlowska, W.; Rutkowski, B. Triple pharmacological blockade of the renin-angiotensin-aldosterone system in nondiabetic CKD: An open-label crossover randomized controlled trial. Am. J. Kidney Dis. 2008, 52, 486-493. [CrossRef] [PubMed]

17. Wang, W.; Li, L.; Zhou, Z.; Gao, J.; Sun, Y. Effect of spironolactone combined with angiotensin converting enzyme inhibitors and/or angiotensin II receptor blockers on chronic glomerular disease. Exp. Ther. Med. 2013, 6, 1527-1531. [CrossRef] [PubMed]

18. Currie, G.; Taylor, A.H.; Fujita, T.; Ohtsu, H.; Lindhardt, M.; Rossing, P.; Boesby, L.; Edwards, N.C.; Ferro, C.J.; Townend, J.N.; et al. Effect of mineralocorticoid receptor antagonists on proteinuria and progression of chronic kidney disease: A systematic review and meta-analysis. BMC Nephrol. 2016, 17, 127. [CrossRef] [PubMed]

19. Bakris, G.L.; Agarwal, R.; Chan, J.C.; Cooper, M.E.; Gansevoort, R.T.; Haller, H.; Remuzzi, G.; Rossing, P.; Schmieder, R.E.; Nowack, C.; et al. Effect of finerenone on albuminuria in patients with diabetic nephropathy: A randomized clinical trial. JAMA 2015, 314, 884-894. [CrossRef] [PubMed]

20. Taira, M.; Toba, H.; Murakami, M.; Iga, I.; Serizawa, R.; Murata, S.; Kobara, M.; Nakata, T. Spironolactone exhibits direct renoprotective effects and inhibits renal renin-angiotensin-aldosterone system in diabetic rats. Eur. J. Pharmacol. 2008, 589, 264-271. [CrossRef] [PubMed]

21. Rocha, R.; Rudolph, A.E.; Frierdich, G.E.; Nachowiak, D.A.; Kekec, B.K.; Blomme, E.A.; McMahon, E.G.; Delyani, J.A. Aldosterone induces a vascular inflammatory phenotype in the rat heart. Am. J. Physiol. Heart Circ. Physiol. 2002, 283, H1802-H1810. [CrossRef] [PubMed]

22. Terada, Y.; Ueda, S.; Hamada, K.; Shimamura, Y.; Ogata, K.; Inoue, K.; Taniguchi, Y.; Kagawa, T.; Horino, T.; Takao, T. Aldosterone stimulates nuclear factor-kappa $\mathrm{b}$ activity and transcription of intercellular adhesion molecule- 1 and connective tissue growth factor in rat mesangial cells via serum- and glucocorticoid-inducible protein kinase-1. Clin. Exp. Nephrol. 2012, 16, 81-88. [CrossRef] [PubMed]

23. Bauersachs, J.; Jaisser, F.; Toto, R. Mineralocorticoid receptor activation and mineralocorticoid receptor antagonist treatment in cardiac and renal diseases. Hypertension 2015, 65, 257-263. [CrossRef] [PubMed]

24. Queisser, N.; Amann, K.; Hey, V.; Habib, S.L.; Schupp, N. Blood pressure has only minor influence on aldosterone-induced oxidative stress and DNA damage in vivo. Free Radic. Biol. Med. 2013, 54, 17-25. [CrossRef] [PubMed]

25. Rocha, R.; Stier, C.T., Jr.; Kifor, I.; Ochoa-Maya, M.R.; Rennke, H.G.; Williams, G.H.; Adler, G.K. Aldosterone: A mediator of myocardial necrosis and renal arteriopathy. Endocrinology 2000, 141, 3871-3878. [CrossRef] [PubMed]

26. Fukuda, S.; Horimai, C.; Harada, K.; Wakamatsu, T.; Fukasawa, H.; Muto, S.; Itai, A.; Hayashi, M. Aldosterone-induced kidney injury is mediated by NFKB activation. Clin. Exp. Nephrol. 2011, 15, 41-49. [CrossRef] [PubMed]

27. Lian, M.; Hewitson, T.D.; Wigg, B.; Samuel, C.S.; Chow, F.; Becker, G.J. Long-term mineralocorticoid receptor blockade ameliorates progression of experimental diabetic renal disease. Nephrol. Dial. Transpl. 2012, 27, 906-912. [CrossRef] [PubMed] 
28. Chen, C.; Liang, W.; Jia, J.; van Goor, H.; Singhal, P.C.; Ding, G. Aldosterone induces apoptosis in rat podocytes: Role of PI3-K/Akt and p38MAPK signaling pathways. Nephron Exp. Nephrol. 2009, 113, e26-e34. [CrossRef] [PubMed]

29. Kobayashi, N.; Hara, K.; Tojo, A.; Onozato, M.L.; Honda, T.; Yoshida, K.; Mita, S.; Nakano, S.; Tsubokou, Y.; Matsuoka, H. Eplerenone shows renoprotective effect by reducing LOX-1-mediated adhesion molecule, PKCepsilon-MAPK-p90RSK, and Rho-kinase pathway. Hypertension 2005, 45, 538-544. [CrossRef] [PubMed]

30. Perez-Rojas, J.M.; Derive, S.; Blanco, J.A.; Cruz, C.; Martínez de la Maza, L.; Gamba, G.; Bobadilla, N.A. Renocortical mRNA expression of vasoactive factors during spironolactone protective effect in chronic cyclosporine nephrotoxicity. Am. J. Physiol. Ren. Physiol. 2005, 289, F1020-F1030. [CrossRef] [PubMed]

31. Luther, J.M.; Luo, P.; Wang, Z.; Cohen, S.E.; Kim, H.S.; Fogo, A.B.; Brown, N.J. Aldosterone deficiency and mineralocorticoid receptor antagonism prevent angiotensin ii-induced cardiac, renal, and vascular injury. Kidney Int. 2012, 82, 643-651. [CrossRef] [PubMed]

32. Nagase, M.; Shibata, S.; Yoshida, S.; Nagase, T.; Gotoda, T.; Fujita, T. Podocyte injury underlies the glomerulopathy of Dahl salt-hypertensive rats and is reversed by aldosterone blocker. Hypertension 2006, 47, 1084-1093. [CrossRef] [PubMed]

33. Nemeth, Z.; Kokeny, G.; Godo, M.; Mózes, M.; Rosivall, L.; Gross, M.L.; Ritz, E.; Hamar, P. Increased renoprotection with ACE inhibitor plus aldosterone antagonist as compared to monotherapies-The effect on podocytes. Nephrol. Dial. Transpl. 2009, 24, 3640-3651. [CrossRef] [PubMed]

34. Tseng, W.C.; Liu, J.S.; Hung, S.C.; Kuo, K.L.; Chen, Y.H.; Tarng, D.C.; Hsu, C.C. Effect of spironolactone on the risks of mortality and hospitalization for heart failure in pre-dialysis advanced chronic kidney disease: A nationwide population-based study. Int. J. Cardiol. 2017, 238, 72-78. [CrossRef] [PubMed]

35. Benson, K.; Hartz, A.J. A comparison of observational studies and randomized, controlled trials. N. Engl. J. Med. 2000, 342, 1878-1886. [CrossRef] [PubMed]

(C) 2018 by the authors. Licensee MDPI, Basel, Switzerland. This article is an open access article distributed under the terms and conditions of the Creative Commons Attribution (CC BY) license (http:/ / creativecommons.org/licenses/by/4.0/). 